\section{Granulicatella spp.}

\author{
César Reyes y M. Elizabeth Barthel
}

Granulicatella spp. is a bacteria of the oral cavity, belonging to the nutritionally variant group streptococci, and has been identified in 5\% of all bacterial endocarditis. It's an important etiologic species in endocarditis, particularly in the setting of negative blood cultures. Granulicatella is a non-mobile, non- spore forming organism that is both catalase and oxidase negative. The treatment for Granulicatella, is the same for Enterococcus according to the American and European guidelines, however resistance to this treatment has been reported.

Key words: Granulicatella, nutritionally variant streptococci, endocarditis.

Palabras clave: Granulicatella, Streptococcus de variante nutricional, endocarditis.

$\mathrm{E}$ n los últimos años, la epidemiología de los microorganismos ha ido variando de la mano de los avances en las técnicas de diagnóstico y clasificación bacteriológica. El advenimiento de técnicas como la reacción de polimerasa en cadena (RPC), replicación de ADN y medios selectivos han permitido descubrir la presencia de nuevos microorganismos que se han identificado como responsables de diversas patologías infecciosas que antes se adjudicaban a los grupos más clásicos y frecuentes. Es así, por ejemplo, que en la endocarditis infecciosa (EI) el microorganismo más frecuentemente aislado es Streptococcus viridans, en $44 \%$ de los casos ${ }^{1}$, sin embargo, existe un porcentaje no despreciable de microorganismos no típicos que han sido identificados como agentes etiológicos de esta patología, siendo Streptococcus de variante nutricional (EVN) responsables de 5\% de los casos con diagnóstico bacteriológico ${ }^{2}$. Este grupo fue descrito por primera vez en 1961 por Frenkel y Hirsh ${ }^{3}$. Treinta y cuatro años después, se estableció a Abiotrophia como un nuevo género del EVN ${ }^{4}$. Posteriormente, la especie Abiotrophia fue reclasificada como Granulicatella, siendo la especie elegans una de las descritas inicialmente ${ }^{5-6}$.

Granulicatella y Abiotrophia son parte de la microbiota normal de la mucosa oral y urogenital, además de la intestinal ${ }^{2}$. Se estima que $G$. elegans está presente en $10 \%$ de la placa dental y puede causar bacteriemia en casos de patología gingival ${ }^{7}$. Una de las características de G. elegans es la producción de actividad bacteriolítica y exopolisacárido ${ }^{7}$. Granulicatella es un organismo no móvil, no formador de esporas, catalasa y oxidasa negativa. Crece como colonias satélite adyacentes a Staphylococcus epidermidis en agar sangre con $\alpha$ hemólisis ${ }^{8}$. Su identificación y diferenciación con Abiotrophia es usualmente hecha con pruebas bioquímicas o por confirmación molecular ${ }^{9,10}$. La espectrometría de masa ${ }^{11}$, si bien es útil, no está ampliamente disponible.

Hospital Naval Almirante Nef, Viña del Mar.

Medicina Interna, Unidad de Cuidados Intensivos (CR). Infectología (MEB).

Recibido: 15 de marzo de 2015 / Aceptado: 7 de abril de 2015

Correspondencia a:

Dr. César Reyes A.

dr.cesar.reyes@gmail.com
Diversas comunicaciones han vinculado a Granulicatella spp. como responsable de diversos cuadros clínicos que difieren de su presentación habitual. Es así como existen datos de que en el grupo de las EI causadas por NVS existe una alta negatividad de los cultivos (41\%), recaídas mayores a las habituales (17\%), y una mortalidad de $17 \%{ }^{12}$. Sin embargo, en los últimos reportes esa cifra ha descendido a $9 \%{ }^{13}$, básicamente por la mayor disponibilidad de técnicas moleculares que permite identificar al microorganismo en las vegetaciones y así dirigir adecuadamente la terapia ${ }^{5}$. Habitualmente la historia de EI por NVS está precedida de un procedimiento dental meses antes, con o sin profilaxis antibacteriana. En total, diecisiete casos de EI por Granulicatella han sido descritos en la literatura científica desde 1997, incluyendo válvulas protésicas y marcapasos. Granulicatella adiacens es el microorganismo más frecuentemente aislado, siendo $G$. elegans un hallazgo infrecuente ${ }^{10}$.

Si bien la EI es la presentación clínica no odontológica más frecuente, también se ha asociado a bacteriemias no asociadas a endocarditis ${ }^{10,14-16}$, sepsis neonatal por transmisión en canal del parto ${ }^{17}$, infección de prótesis aórtica abdominal ${ }^{9}$, infección de catéter tunelizado para hemodiálisis tipo Tesio $^{9}$, absceso cerebral ${ }^{18}$, meningitis ${ }^{19}$, artritis séptica ${ }^{20}$, osteomielitis vertebral $^{21,22}$, prótesis mamaria ${ }^{23} \mathrm{y}$ catéter de peritoneo diálisis ${ }^{24}$.

En cuanto al estudio de susceptibilidad, la recomendación es no realizarla mediante difusión en disco, sino que por método de microdilución ${ }^{25}$. Los hallazgos en la literatura reportan una susceptibilidad de $60 \%$ para penicilina G, $60 \%$ para ceftriaxona, $81 \%$ para amoxicilina y $96 \%$ para meropenem en caso de G. adiacens. Granulicatella elegans es $100 \%$ sensible a penicilina y $33 \%$ a ceftriaxona ${ }^{26,27}$.

De acuerdo a la experiencia descrita en las publicaciones médicas, los antibacterianos más frecuentemente usados han sido penicilina $\mathrm{G}$, amoxicilina y gentamicina. Las guías de la American Heart Association recomiendan que las EI por EVN deben ser tratadas con un esquema activo frente a Enterococcus spp. ${ }^{28}$. La British Society for Antimicrobial Chemotherapy hace la misma recomendación ${ }^{29}$. Se sugiere un tratamiento extendido por seis semanas, existiendo algunos casos agresivos con necesidad de cirugía de reemplazo valvular. La recaída es frecuente ${ }^{12}$, al igual que la insuficiencia cardíaca asociada y la embolización hacia territorio distal ${ }^{30}$. La mortalidad global es de 9,5\%; dada principalmente por los casos de EI.

En resumen, los EVN corresponden a un grupo de microorganismos que en las últimas décadas han emergido como responsables de enfermedades importantes como la EI, siendo necesaria su consideración al momento de estudiar una EI con cultivo negativo o en una identificación inicial como Abiotrophia spp. Se deben utilizar técnicas de diagnóstico microbiológico automatizado y moleculares, así como evitar el método de difusión en disco para determinar su susceptibilidad. Se recomienda el tratamiento con $\beta$ lactámicos por un período de seis semanas.

\section{Referencias bibliográficas}

1.- Tleyjeh I M, Steckelberg J M, Murad H S, Anavekar N S, Ghomrawi H M, Mirzoyev Z, et al. Temporal trends in infective endocarditis: a population-based study in Olmsted County, Minnesota. JAMA 2005; 293: 3022-8.

2.- Rouff K L. Nutritionally variant streptococci. Clin Microbiol Rev 1991; 4: 184-90.

3.- Frenkel A, Hirsch W. Spontaneous development of L forms of streptococci requiring secretions of other bacteria or sulphydryl compounds for normal growth. Nature 1961; 191: 728-30. 
4.- Kawamura Y, Hou X-G, Sultana F, Liu S, Yamamoto H, Ezaki T. Transfer of Streptococcus adjacens and Streptococcus defectivus to Abiotrophia gen. nov. as Abiotrophia adiacens comb. nov. and Abiotrophia defectiva comb. nov., respectively. Int J Syst Bacteriol 1995; 45: 798-803.

5.- Casalta J P, Habib G, La Scola B, Drancourt M, Caus T, Raoult D. Molecular diagnosis of Granulicatella elegans on the cardiac valve of a patient with culture-negative endocarditis. J Clin Microbiol 2002; 40: 1845-7.

6.- Ruoff K L. Aerococcus, Abiotrophia, and other aerobic catalase-negative, gram-positive cocci. Murray PR, Baron E J, J. J. H., Landry M L, Pfaller M A, editors. Manual of Clinical Microbiology, 9th ed. Washington, DC: ASM Press 2007; p. 443-54.

7.- Ohara-Nemoto Y, Kishi K, Satho M, Tajika S, Sasaki M, Namioka A, et al. Infective endocarditis caused by Granulicatella elegans originating in the oral cavity. J Clin Microbiol 2005; 43: 1405-7.

8.- Roggenkamp A, Abele-Horn M, Trebesius K H, Tretter U, Autenrieth I B, Heesemann J. Abiotrophia elegans sp. nov. a possible pathogen in patient with culture-negative endocarditis. J Clin Microbiol 1998; 36: 100-4.

9.- Cargill J S, Scott K S, Gascoyne-Binzi D, Sandoe J A. Granulicatella infection: diagnosis and management. J Med Microbiol 2012; 61: 755-61.

10.- Christensen J J, Facklam R R. Granulicatella and Abiotrophia species from human clinical specimens. J Clin Microbiol 2001; 39: 3520-3.

11.- Ratcliffe P, Fang H, Thidholm E, Borang S, Westling K, Ozenci B. Comparison of MALDI-TOF MS and VITEK 2 system for laboratory diagnosis of Granulicatella and Abiotrophia species causing invasive infections. Diagn Microbiol Infect Dis 2013; 77: 216-9.

12.- Stein D S, Nelson K E. Endocarditis due to nutritionally deficient streptococci: therapeutic dilemma. Rev Infect Dis 1987; 9: 908-16.

13.- Giuliano S, Caccese R, Carfagna P, Vena A, Falcone M, Venditti M. Endocarditis caused by nutritionally variant streptococci: a case report and literature review. Infez Med 2012; 20: 67-74.

14.- Abdul-Redha R J, Prag J, Sonksen U W, Kemp M, Andresen K, Christensen J J. Granulicatella elegans bacteremia in patients with abdominal infections. Scand J Infect Dis 2007; 39; 830-3.

15.- Senn L, Entenza J M, Greub G, Jaton K, Wenger A, Bille J, et al. Bloodstream and endovascular infections due to Abiotrophia defectiva and Granulicatella species. BMC Infect Dis 2006; 6: 9.

16.- Woo P C, Fung A M, Lau S K, Chan B Y, Chiu S K, Teng J L. Granulicatella adiacens and Abiotrophia defective bacteraemia characterized by $16 \mathrm{~S}$ rRNA gene sequencing. J Med Microbiol 2003; 52: 137-40.

17.- Bizzarro M J, Callan D A, Farrel P A, Dembry L M, Gallagher P G. Granulicatella adiacens and early-onset sepsis in neonate. Emerg Infect Dis 2011; 17: 1971-3.

18.- Biermann C, Fries G, Jehnichen P, Bhakdi S, Husmann M. Isolation of
Abiotrophia adiacens from a brain abscess which developed in a patient after neurosurgery. J Clin Microbiol 1999; 37: 769-71.

19.- Cerceo E, Christie J D, Nachamkin I, Lautenbach E. Central nervous system infections due to Abiotrophia and Granulicatella species: an emerging challenge? Diagn Microbiol Infect Dis 2004; 48: 161-5.

20.- Hepburn M J, Fraser S L, Rennie T A, Singleton C M, Delgado B Jr. Septic arthritis caused by Granulicatella adiacens: diagnosis by inoculation of synovial fluid into blood culture bottles. Rheumatol Int 2003; 23: 255-7.

21.- Rosenthal O, Woywodt A, Kirschner P, Haller H. Vertebral osteomyelitis and endocarditis of a pacemaker lead due to Granulicatella (Abiotrophia) adiacens. Infection 2002; 30: 317-9.

22.- Fukuda R, Oki M, Ueda A, Yanagi H, Komatsu M, Itoh M, et al. Vertebral osteomyelitis associated with Granulicatella adiacens. Tokai J Exp Clin Med 2010; 35: 126-9.

23.- del Pozo J L, García-Quetglas E, Hernaez S, Serrera A, Alonso M, Pina L, et al. Granulicatella adiacens breast implant-associated infection. Diagn Microbiol Infect Dis 2008; 61: 58-60.

24.- Altay M, Akay H, Yildiz E, Duranay M. A novel agent of peritoneal dialysis-related peritonitis: Granulicatella adiacens. Perit Dial Int 2008; 28: 96-7.

25.- Jorgensen J H, Hindler J F. New consensus guidelines from the Clinical and Laboratory Standards Institute for antimicrobial susceptibility testing of infrequently isolated or fastidious bacteria. Clin Infect Dis 2007; 44: 280-6.

26.- Tuohy M J, Procop G W, Washington J A. Antimicrobial susceptibility of Abiotrophia adiacens and Abiotrophia defectiva. Diagn Microbiol Infect Dis 2000; 38: 189-91.

27.- Zheng X, Freeman A F, Villafranca J, Shortridge D, Beyer J, Kabat W, et al. Antimicrobial susceptibilities of invasive pediatric Abiotrophia and Granulicatella isolates. J Clin Microbiol 2004; 42: 4323-6.

28.- Baddour L M, Wilson W R, Bayer A S, Fowler V G Jr, Bolger A F, Levison M E, et al. Infective endocarditis: diagnosis, antimicrobial therapy, and management of complications: a statement for healthcare professionals from the Committee on Rheumatic Fever, Endocarditis, and Kawasaki Disease, Council on Cardiovascular Disease in the Young, and the Councils on Clinical Cardiology, Stroke, and Cardiovascular Surgery and Anesthesia, American Heart Association: endorsed by the Infectious Diseases Society of America. Circulation 2005; 111: e394-434.

29.- Elliott T S J, Foweraker J, Gould F K, Perry J D, Sandoe J A. Guidelines for the antibiotic treatment of endocarditis in adults: report of the Working Party of the British Society for Antimicrobial Chemotherapy. J Antimicrob Chemother 2004; 54: 971-81.

30.- Lin C H, Hsu R B. Infective endocarditis caused by nutritionally variant streptococci. Am J Med Sci 2007; 334: 235-9. 